\title{
Software Verification with Liquid Types ${ }^{\star}$
}

\author{
Ranjit Jhala \\ University of California, San Diego \\ jhala@cs.ucsd.edu
}

\begin{abstract}
Traditional software verification algorithms work by using a combination of Floyd-Hoare Logics, Model Checking and Abstract Interpretation, to check and infer suitable program invariants. However, these techniques are problematic in the presence of complex but ubiquitous constructs like generic data structures, first-class functions. We observe that modern type systems are capable of the kind of analysis needed to analyze the above constructs, and we use this observation to develop Liquid Types, a new static verification technique which combines the complementary strengths of Floyd-Hoare logics, Model Checking, and Types. As a result, we demonstrate how liquid types can be used to statically verify properties ranging from memory safety to data structure correctness, in higher-order languages like ML. This presentation is based on joint work with Patrick Rondon and Ming Kawaguchi.
\end{abstract}

\footnotetext{
* This work was supported by NSF grants CCF-0644361, CNS-0720802, CCF-0702603, and gifts from Microsoft Research.
} 